\title{
A question of benefit
}

\section{Newborn screening panels for genetic diseases are now nearly uniform across the United States, and expanded panels now test for diseases for which there is no known treatment. This expansion of newborn screening raises questions about whether traditional assumptions of implied consent are appropriate.}

$T_{\text {in }}^{\text {he }}$ here have been substantial changes in newborn screening programs in the United States in the past six years, the time elapsed since our 2004 editorial entitled "Separated at birth" (Nat. Genet. 36, 1127, 2004). That editorial noted the inconsistency of newborn screening panels between states and endorsed the American College of Medical Genetics recommendation of a uniform mandatory newborn screening panel of 29 core conditions. Currently, all states and the District of Columbia screen for almost all of the core conditions on the recommended panel, with the exception of severe combined immunodeficiency (SCID), which was only recently added. Notably, the SCID test is the first DNAbased test and as such marks an expansion of newborn screening into the DNA-testing arena.

Historically, newborn screening was intended to identify serious disorders in which there is a known benefit to presymptomatic diagnosis and treatment. The classic example is phenylketonuria, for which early detection allows for immediate dietary intervention and prevention of neurological harm from elevated levels of phenylalanine. The justification for the broad public health scope of newborn screening in which consent is implied and participation can be mandatory was that there is an immediate benefit to the newborn.

There have been a number of departures from this original paradigm in the recent expansion of newborn screening and with the advent of tandem mass spectrometry as the testing method. First, the concept of benefit has been broadened to justify testing for conditions with no known cure but for which there are treatment options that improve the prognosis. An example is cystic fibrosis, for which early intervention with nutritional therapies can improve growth, reduce hospitalizations and increase life expectancy.

Second, tandem mass spectrometry can incidentally plain identify diseases that are not on the recommended panel and for which there is no known treatment. The benefits used to justify revealing such results to parents include the value of receiving a diagnosis and preempting the 'diagnostic odyssey', and the benefit of the timely provision of information to parents about reproductive risk for family planning decisions. There is also a potential societal benefit in that early identification of individuals with these conditions provides an opportunity for the medical community to learn about the natural history of the disease.

A third issue is the use of newborn screening tests to identify individuals who are carriers for one of the conditions on the panel. With current technology, this is possible for both sickle cell anemia and cystic fibrosis. Although there may be long-term medical benefits of notifying carriers who may be at increased risk for rare complications, the primary benefit used to justify carrier status notification is that this information may be of value to the individual, the parents and other family members for purposes of reproductive risk assessment.

It is easy to see the argument for the value of these expanded notions of benefit. Indeed, some of the strongest voices supporting expansion of newborn screening beyond the 'screen for it only if you can treat it' paradigm are advocacy organizations representing parents with affected children. These organizations almost always support expanded screening programs because it is clear that a majority of parents place a high value on a diagnosis even if there is no treatment.

However, for genetic tests with a primary goal of obtaining information on reproductive risk, such as prenatal genetic testing and preconception genetic testing, ethical norms dictate that testing be voluntary and performed within a system providing access to genetic counseling. As pointed out by Yvonne Bombard and colleagues in Nature Reviews Genetics, there are no currently accepted ethical guidelines for mandatory screening and disclosure when the benefit is reproductive risk information (Nat. Rev. Genet. 10, 666-667, 2009).

Newborn screening is an extraordinarily successful example of the application of advances in medical genetics to personal and public health benefit. The evidence-based method of evaluating conditions for inclusion on newborn screening panels, developed by the US Department of Health and Human Services Secretary's Advisory Committee on Heritable Disorders in Newborns and Children and described in a recent special focus on newborn screening in Genetics in Medicine, has laudably made the process rigorous and transparent. However, the purpose and practice of newborn screening needs careful consideration and vetting by stakeholders in the public health, medical genetics, advocacy, and ethical, legal and social issues communities. It seems clear that new practices have moved beyond the original intent of newborn screening, and it needs to be determined if future expansions of newborn screening incorporating these broadened concepts of benefit can be accommodated within the original paradigm in which consent is implied. It may be necessary to envision an alternative screening program based on voluntary participation and informed consent that could accommodate these expanding notions of benefit.

The interested parties should take into account the changing landscape of our society in which individuals are seeking and receiving personal genetic information through direct-to-consumer genetic testing, pharmacogenomics and personal genome sequencing. Does the uptake of these new technologies by the public mean that more people than previously anticipated are ready to make sense of personal genomic information? More likely, it highlights the value of the opportunity to educate the public about genetics that is afforded by newborn screening at every birth. 